\title{
Sulindac-Induced Stevens-Johnson Toxic Epidermal Necrolysis Syndrome: Case Report
}

\author{
Aguilar Silva $\mathrm{A}^{1 *}$, Espinosa Tavitas $\mathrm{M}^{2}$, Carpio Orantes $\mathrm{LD}^{3}$, Lopez Cabrera \\ $\mathbf{Y}^{1}$, Mayo Martinez ML $^{1}$ and Guerrero Martinez MD $^{1}$ \\ ${ }^{1}$ Resident physician. Department of Internal Medicine, “Adolfo Ruiz Cortines” National Medical \\ Center, Instituto Mexicano del Seguro Social, Mexico \\ ${ }^{2}$ Dermatologist, Professor at Universidad Veracruzana México \\ ${ }^{3}$ Department of internal medicine, General hospital 71, Instituto Mexicano del Seguro Social, \\ Mexico
}

*Corresponding author: Andres Aguilar, Resident physician. Department of Internal Medicine, "Adolfo Ruiz Cortines" National Medical Center, Instituto Mexicano del Seguro Social, Mexico; Email: andyelguero@hotmail.com

\section{Abstract}

We present the case of a 42-year-old patient who came to the emergency room after taking sulindac as a treatment for diabetic neuropathy, presenting 4 days later a maculo-violaceous rash on the chest accompanied by vesicles, pruritus, extending to the abdomen and upper extremities and lower lesions and fever, affecting approximately $25 \%$ of the body surface, basophil degranulation was performed, reporting $50 \%$ for sulindac. A literature review was carried out in order to update the current knowledge about Steven Johnson syndrome in the hospital environment, etiology, clinical manifestations, epidemiology, diagnosis and treatment.

Keywords: Sulindac; Steven Johnson syndrome; Toxic epidermal necrolysis

\section{Introduction}

Adverse skin reactions to medications are common unwanted effects, most of which are mild and can rarely be fatal. The prevalence is approximately 1-3\% in hospitalized patients. (1) $2-5 \%$ of adverse skin reactions to drugs are considered severe (Severe Cutaneous Adverse Reactions [SCAR]), within this group is the syndrome of Steven Johnson and Toxic Epidermal Necrolysis [1].

Steven Johnson syndrome (SJS) and toxic epidermal necrolysis (TEN) are rare, life-threatening disorders characterized by generalized mucosal and epidermal necrosis. Operationally, they can be distinguished by virtue of the fact that SJS tends to involve $10 \%$ of the body surface area, while in TEN it is affected from one third to $100 \%$, just as there is overlap SSJ and NET when there is a $10-30 \%$ involvement [2].

\section{Background}

In 1922 it was first described by two American physicians in two children with a generalized exanthematic eruption associated with maculae with a necrotic center, purulent conjunctivitis, severe stomatitis, and fever. In 1956, 34 years later, the dermatologist Alan Lyell reported a series of cases with a mucocutaneous reaction with necrosis of the skin, which he called toxic epidermal necrolysis or Lyell syndrome. "Necrolysis" due to the combination of epidermolysis with the histopathological characteristic of necrosis and "toxic" as he suspected it was due to a toxin $[3,4]$.

\section{Epidemiology}

In Mexico we do not have data indicating the number of annual cases. We have large cohorts such as that of the EUROSCAR registry, a multinational study carried out in 
Europe from 1997-2001 which reported severe adverse skin reactions through a network of hospitals that covers more than 100 million inhabitants, which report the annual incidence for Stevens-Johnson syndrome and for toxic epidermal necrolysis between 1.2 and 6 and between 0.5 and 1.2 cases per million inhabitants, respectively [5].

\section{Etiology}

Medications are the main trigger for Stevens-Johnson syndrome / toxic epidermal necrolysis (SJS / TEN) in both adults and children. The risk of SJS / TEN appears to be limited to the first eight weeks of treatment. Drugs used longer are unlikely to be the cause of SJS / TEN. The typical exposure period before the onset of the reaction is four days to four weeks from the first continuous use of the drug.

\begin{tabular}{|c|}
\hline Strongly associated \\
\hline Allopurinol \\
\hline Lamotrigine \\
\hline Sulfamethoxazole \\
\hline Carbamazepine \\
\hline Phenytoin \\
\hline Nevirapine \\
\hline Sulfasalazine \\
\hline Oxicam NSAIDs (piroxicam, tenoxicam) \\
\hline Phenobarbital \\
\hline Etoricoxib \\
\hline Associated \\
\hline Diclofenac \\
\hline Doxycycline \\
\hline Amoxicillin / Ampicillin \\
\hline Ciprofloxacin \\
\hline Levofloxacin \\
\hline Amifostine \\
\hline Oxcarbazepine \\
\hline Rifampicin \\
\hline Suspicious Association / Low Risk \\
\hline Pantoprazole \\
\hline Glucocorticoids \\
\hline Omeprazole \\
\hline Tetrazepam \\
\hline Dipyrone (Metamizole) \\
\hline Terbinafine \\
\hline Levetiracetam \\
\hline
\end{tabular}

Table 1: Drugs associated with Steven Johnson Syndrome / Toxic Epidermal Necrolysis (SJS / TEN).
In the last 20 years, two large multinational case control studies, published in 1995 and 2008, identified different degrees of drug association with SJS / TEN: 'strongly associated', 'associated', 'suspect' and 'non-suspect' [6] (Table 1).

Infection is the second most common precipitating cause. The main germs involved are: Mycoplasma pneumoniae, Cytomegalovirus, Herpes virus and Hepatitis A virus [7]. In more than a third of SJS / TEN cases, no cause can be identified. Rare and debatable causes of SJS / TEN include vaccines, systemic diseases, contrast medium, and chemicals in food [8-10].

Risk factors: Adulthood: between 20 and 49 years old, female sex, recurrent exposure to the same drugs [11]. Strong associations between drugs and specific HLA alleles have been reported in Asian populations such as HLAB * 15: 02 and HLA B * 58: 01 that are strongly associated with SJS / NET induced by Carbamazepine and Allopurinol respectively [12].

The incidence of SJS / TEN is 1000 times higher in patients with HIV. The high incidence in these patients may be due to polypharmacy, slow acetylation of drugs, altered lymphocyte function, and cytotoxic metabolites in the case of trimethoprim / sulfamethoxazole [13]. An association with immunosuppressive states, epilepsy, depression, diseases of collagen such as systemic lupus erythematosus, pneumonia, chronic kidney disease, cancer (mainly of hematological origin) $[14,15]$.

\section{Pathophysiology}

In the case of SJS and TEN, with the response to drugs the causal mechanism is of an adaptive immune type due to a delayed IV hypersensitivity response according to the Gell and Coombs classification [16]. It has been cited as an abnormal immune response of some individuals to certain drugs, including lamotrigine, which causes a type IV hypersensitivity reaction, which is mediated by cells in which CD8 + and CD4 + T lymphocytes release cytotoxic mediators resulting in programmed cell death (apoptosis) of keratinocytes [17].

\section{Clinical Manifestations}

Typically begins with a febrile picture, often above $39^{\circ} \mathrm{C}$, and flu-like symptoms precede the development of mucocutaneous lesions by one or three days. Some symptoms such as photophobia, conjunctival itching or burning, dysphagia, general malaise, myalgias and arthralgias are very common [18]. The lesions begin on the trunk, with a painful rash those progresses rapidly with posterior involvement 


\section{Clinical Dermatology Open Access Journal}

of the neck, face, and upper extremities, in its proximal portion, with a bilateral and symmetrical arrangement. Usually the distal portions of the extremities are spared, with little involvement of the palms and soles. Nikolsky's sign (superficial detachment by applying gentle pressure to the surface of the skin) can be positive. Oral, ocular and / or genital mucositis with painful mucosal erosions. AsboeHansen's sign or "bulla extension sign" (a lateral extension of the bulla with pressure) may also be present $[19,20]$.

\section{Diagnosis}

There are no universally accepted diagnostic criteria for Stevens-Johnson syndrome / toxic epidermal necrolysis (SJS / TEN), and the histological findings are neither specific nor diagnostic. Despite these limitations, the diagnosis of SJS or TEN would be appropriate in a patient with the following clinical characteristics:

Suggestive history of drug exposure; Exposure to the drug usually precedes the onset of symptoms by one to four weeks (average 14 days), but re-exposure can cause symptoms to appear in as little as 48 hours, prodrome of disease, and acute-onset febrile malaise.

Potentially aggressive drugs can be targeted based on Alden's six parameters (drug causation algorithm for epidermal necrolysis): time delay from drug administration to onset of reaction, probability of drug presence in body, previous exposure to the same drug regardless of the reaction at that time, presence of the drug beyond the progression phase, notoriety of the drug as a cause of SJS / TEN, and presence or absence of other etiologies. The symptoms of SJS / TEN are not clearly attributed to a drug in $20 \%$ to $25 \%$ of cases [21].

Skin biopsy is helpful in confirming the diagnosis and excluding other conditions that may mimic SJS / TEN [22].

The modified DB test is useful for the diagnosis of type I hypersensitivity, the mechanism most frequently implicated [23].

\section{Treatment}

Discontinuation of the causative agent since delayed suspension is associated with an increase in mortality, supportive treatment with a correct intake of fluids and electrolytes, nutritional support and management of body temperature, in addition to the management of infections or other complications that can be presented. It is recommended that the hospitalization be carried out in isolation conditions that allow the monitoring and prevention of infections $[24,25]$. Frequent application of emollients to the skin is useful during the acute phase to support barrier function, reduce transcutaneous water loss, and promote re-epithelialization. The surgical approach involves debridement of the detached epidermis to remove potentially infected material followed by physiological closure of the wounds using biosynthetic dressings. Among the different systemic immunomodulatory therapies, glucocorticosteroids and cyclosporine are the most promising in the treatment of SJS / NET. However, more studies are required to validate this conclusion [26].

\section{Prognosis}

The prognosis of individual patients can be quickly assessed in the early stages of the disease by applying a prognostic scoring system called SCORTEN (Table 2) [27], based on seven clinical and laboratory variables that must be calculated in the early stages. 24 hours after the patient's hospitalization. The estimated mortality rate is as high as 30 to $50 \%$ for toxic epidermal necrolysis and less (5\%) for Stevens-Johnson syndrome [28] (Table 2).

\begin{tabular}{|c|c|c|}
\hline \multicolumn{2}{|c|}{ Independent risk factors Weight } & Independent risk factors Weight \\
\hline Age Over 40 years & Age Over 40 years & Age Over 40 years \\
\hline Malignancy Yes & Malignancy Yes & Malignancy Yes \\
\hline Body surface area Greater than $10 \%$ & Body surface area Greater than $10 \%$ & Body surface area Greater than $10 \%$ \\
\hline Tachycardia Greater than 120 bpm & Tachycardia Greater than 120 bpm & Tachycardia Greater than 120 bpm \\
\hline Serum urea Greater than $10 \mathrm{mmol} / \mathrm{L}$ & Serum urea Greater than $10 \mathrm{mmol} / \mathrm{L}$ & Serum urea Greater than $10 \mathrm{mmol} / \mathrm{L}$ \\
\hline \multicolumn{3}{|c|}{ Serum glucose Greater than 14 mmol / $\mathbf{~}$ Serum glucose Greater than $14 \mathrm{mmol} / \mathrm{L}$ Serum glucose Greater than $14 \mathrm{mmol} / \mathrm{L}$} \\
\hline Seric bicarbonate Less than $20 \mathrm{mmol} / \mathrm{L}$ & Seric bicarbonate Less than $20 \mathrm{mmol} / \mathrm{L}$ & Seric bicarbonate Less than $20 \mathrm{mmol} / \mathrm{L}$ \\
\hline \multicolumn{2}{|c|}{ SCORTEN 7} & SCORTEN 7 \\
\hline
\end{tabular}

Table 2: SCORTEN score for Stevens-Johnson syndrome / toxic epidermal necrolysis. 


\section{Clinical Case}

42-year-old male patient with a history of type 2 diabetes Mellitus, 12 years of evolution in treatment with insulin glargine 20 IU every 24 hours at night, metformin every 8 hours, denies SAH, refers to having presented chancre on the penis ago 6 years the etiology is unknown, diagnosis of diabetic neuropathy, so on 10/31/19 he goes to a family doctor who prescribes Sulindaco $200 \mathrm{mg}$ every 24 hours for 10 days and carbamazepine 200 mg every 24 hours for 30 days, the patient starts treatment that day; At 2 days, he began with general malaise and fever of $38^{\circ}$, the next day he presented confluent violaceous maculopapular lesions in the chest accompanied by itching, which spread to the abdomen and upper and lower limbs, on the fourth day after the start of taking The medications go to a doctor, who requests laboratory studies to rule out dengue, and starts paracetamol. On the fifth day, he comes to the emergency room with desquamation of the mucosa of the penis and glans, testicular edema and cracked lip corners that causes bleeding; as well as violaceous macules that spread on the palms and soles of the hands, Nikolsky's sign $(+)$, initially laboratory studies are requested which report mild lymphopenia and non-reactive HIV test, viral panel of Hepatitis B, C and Negative VDRL, basophil degranulation to metformin, carbamazepine and sulindac was requested, reporting a $50 \%$ degranulation to the latter, within the physical examination the presence of violaceous macules on the face, chest, abdomen and extremities including palms and soles, with lesions stands out. bullous, with the presence of erythema in the conjunctiva, with cracked lips with slight bleeding, hyperemic pharynx with the presence of red dotting, with whitish plaques on the soft palate, erythematous, edematous tongue, with lymphadenopathy of approximately $3 \mathrm{~cm}$ in English bilaterally, with edema In testicles, with erythema and desquamation of the glans, parenteral hydration was administered, ceftriaxone $1 \mathrm{~g}$ Intravenous every $12 \mathrm{~h}$, diphenhydramine $20 \mathrm{mg}$ intravenously every $8 \mathrm{~h}$, ranitidine 50mg intravenously every $12 \mathrm{~h}$, paracetamol 1 gram orally as analgesic and antipyretic. Antiseptic solutions in oral mucosa, chloramphenicol otic solution 2 drops every 4 hours daily wound washing. With stay for 7 days in hospitalization and discharge due to improvement. The treatment indicated at discharge was almond oil, cold cream and antifungal agents (Figures $1 \& 2$ ) (Table 3).

\begin{tabular}{|c|c|c|c|}
\hline & & \multicolumn{2}{|c|}{ Basophil degranulation } \\
\hline Glucose & 220 & Drug 1: Metformin 850mg & $2 \%$ \\
\hline Creatinine & 0.8 & Drug 2: Sulindaco 200mg & $50 \%$ \\
\hline BT / BD & $0.5 / 0.3$ & Drug 3: Carbamazepine 200mg & $2 \%$ \\
\hline Hb / Leukocytes & $14-\mathrm{Jul}$ & ELISA Test for HIV & Negative \\
\hline Eosinophils Percentage / Total & $3.20 \% / \# 0.23$ & Viral panel for Hepatitis B, C & Negative \\
\hline Basophils Percentage / Total & $1 \% / \# 0.07$ & VDRL & Negative \\
\hline
\end{tabular}

Table 3: Biochemical characteristics of the patient during his hospitalization.

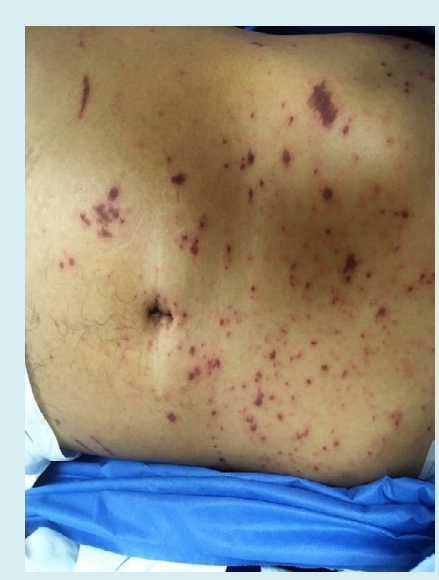

Figure 1: Crusted lesions on the abdomen.

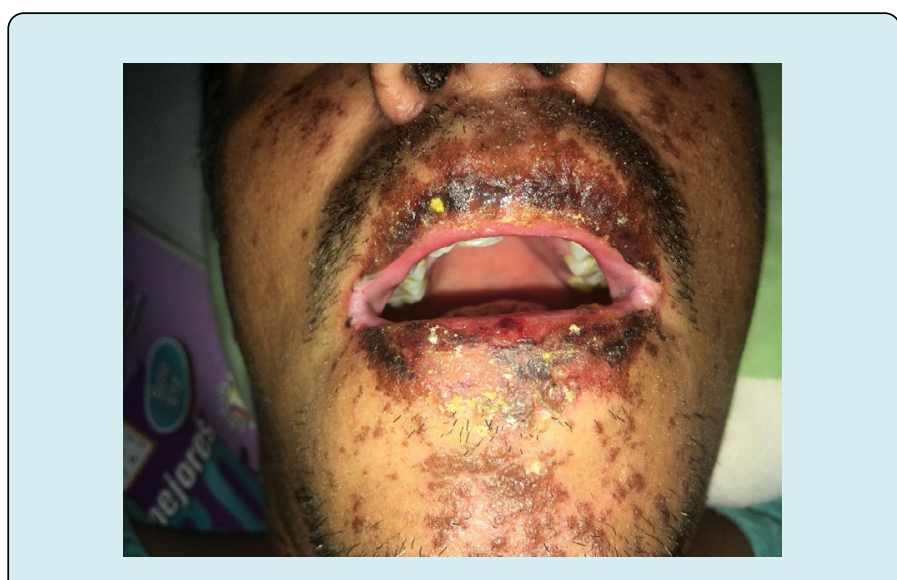

Figure 2: Patient with multiple lesions on the lips and face. 


\section{Clinical Dermatology Open Access Journal}

\section{Discussion}

Sulindac was made available in the United States, in 1978, for use as a non-steroidal anti-inflammatory agent. In 1982, a report was made of four patients who had fever and involvement of one or more organs after therapy with sulindac [29-31], in our case the patient received therapy for diabetic neuropathy with carbamazepine and sulindac, presenting later symptoms. Of the documented medications that the patient had consumed, basophil degranulation was requested for the anticonvulsant carbamazepine, the oral hypoglycemic agent metformin (biguanide) and sulindac from the NSAID group. Reporting positive for sulindac with a response of $50 \%$. Although the basophil degranulation test has a low NPV, it presents high PPV and together with the clinical evaluation, it allows the specialist to arrive at the diagnosis. It is important to make a differential diagnosis in these patients, ruling out vasculitis with cutaneous manifestations, bullous pemphigus, Reiter's syndrome, bullous pemphigus, among others. Treatment is supportive and prevention of complications, with treatment of skin lesions, oral, ophthalmological control.

\section{Conclusion}

Steven Johnson syndrome is a very rare entity, In most cases it is secondary to the use of: antibiotics, anticonvulsants, NSAIDs, among others, Other less frequent causes include bacterial, viral, fungal infections and to a great extent idiopathic, The manifestations begin 4 to 10 days after the use of the drug, The treatment includes withdrawal of the drug, immunoglobulins, steroid management, asepsis of affected regions, adequate intravenous hydration. However, the occurrence of serious adverse events, as reported here, indicates that sulindac should be administered as carefully as other non-steroidal anti-inflammatory drugs.

\section{References}

1. Martínez S, Gómez M, Ocampo J (2015) Actualidades en farmacodermias severas: síndrome de Stevens- Johnson (SSJ) y necrólisis epidérmica tóxica (NET). Gac Med Mex 151(6): 777-787.

2. Burn T, Breathnach S, Griffiths C (2010) Erythema multiforme, Stevens-Johnson syndrome and toxic epidermal necrolysis. Rook's Textbook of Dermatology 76(1): 21-22.

3. Stevens AM, Johnson FC (1922) An eruptive fever associated with stomatitis and ophthalmia. Am J Dis Child 24(6): 526-533.

4. Lyell A (1956) Toxic epidermal necrolysis: An eruption resembling scalding of the skin. Br J Dermatol 68(11):
355-361.

5. Mockenhaupt M, Viboud C, Dunant A, Naldi L, Halevy $S$, et al. (2008) Stevens-Johnson syndrome and toxic epidermal necrolysis: assessment of medication risks with emphasis on recently marketed drugs. The EuroSCAR-study. J Invest Dermatol 128(1): 35-44.

6. Mockenhaupt M (2010) International registry of severe cutaneous adverse reactions (SCAR) to drugs and collection of biological sample, study protocol. RegiSCAR study group 1-21.

7. White KD, Abe R, Ardern-Jones M, Beachkofsky T, Shear NH,etal.(2018)SJS/TEN2017: Building Multidisciplinary Networks to Drive Science and Translation.The Journal of Allergy and Clinical Immunology. The Journal of Allergy and Clinical Immunology: In Practice 6(1): 38-69.

8. Ball R, Ball LK, Wise RP, Braun MM, Beeler JA, et al. (2001) Stevens-Johnson syndrome and toxic epidermal necrolysis after vaccination: reports to the vaccine adverse event reporting system. Pediatr Infect Dis J 20(2): 219-223.

9. Oda T, Sawada Y, Okada E, Yamaguchi T, Haruyama S, et al. (2017) Stevens-Johnson Syndrome After Influenza Vaccine Injection. J Investig Allergol Clin Immunol 27(4): 274-275.

10. Haddad C, Chosidow O, Valeyrie-Allanore L, Ghaleh B, Legrand T, et al. (2017) Are Idiopathic Stevens-Johnson Syndrome/Toxic Epidermal Necrolysis Related to Drugs in Food? The Example of Phenylbutazone. J Invest Dermatol 137(5): 1179-1181.

11. Nguyen DV, Chu HC, Phan MH, Craig T, Baumgart K, et al. (2015) HLA-B*1502 and carbamazepine-induced severe cutaneous adverse drug reactions in Vietnamese. Asia Pacific Allergy 5(2): 68-77.

12. Wang Q Sun S, Xie M, Li X, Zhao Z, et al. (2017) Association between the HLA-B alleles and carbamazepine-induced SJS/TEN: A meta-analysis. Epilepsy Res135: 19-28.

13. Mittmann N, Knowles SR, Koo M, Shear NH, Rachlis A, et al. (2012) Incidence of toxic epidermal necrolysis and Stevens-Johnson Syndrome in an HIV cohort: an observational, retrospective case series study. Am J Clin Dermatol 13(1): 49-54.

14. Frey N, Jossi J, Bodmer M, Bircher A, Jick SS, et al. (2017) The Epidemiology of Stevens- Johnson Syndrome and Toxic Epidermal Necrolysis in the UK. Journal of Investigative Dermatology 137(6): 1240-1247.

15. Martínez S, Gómez M, Ocampo J (2015) Actualidades en 


\section{Clinical Dermatology Open Access Journal}

farmacodermias severas: síndrome de Stevens- Johnson (SSJ) y necrólisis epidérmica tóxica (NET). Gac Med Mex 151(6): 777-787.

16. Ardern-Jones MR, Friedmann PS (2011) Skin manifestations of drug allergy. Br J Clin Pharmacol 71(5): 672-683.

17. Lerch M, Mainetti C, Terziroli Beretta-Piccoli B, Harr $\mathrm{T}$ (2018) Current Perspectives on Stevens-Johnson Syndrome and Toxic Epidermal Necrolysis. Clin Rev Allergy Immunol 54(1): 147-176.

18. Schwartz RA, McDonough PH, Lee BW (2013) Toxic epidermal necrolysis: Part I. Introduction, history, classification, clinical features, systemic manifestations, etiology, and immunopathogenesis. J Am Acad Dermatol 69(3): 173.

19. Valeyrie-Allanore L, Roujeau JC (2012) Epidermal necrolysis (Stevens-Johnson syndrome and toxic epidermal necrolysis). Fitzpatrick's Dermatology in General Medicine, $8^{\text {th }}$ (Edn.), New York, McGraw-Hill, pp: 439.

20. Schwartz RA, McDonough PH, Lee BW (2013) Toxic epidermal necrolysis: Part II. Prognosis, sequelae, diagnosis, differential diagnosis, prevention, and treatment. J Am Acad Dermatol 69(2): 187.

21. Sassolas B, Haddad C, Mockenhaupt M, Dunant A, Liss $Y$, et al. (2010) ALDEN, an algorithm for assessment of drug causality in Stevens-Johnson Syndrome and toxic epidermal necrolysis: comparison with case-control analysis. Clin Pharmacol Ther 88(1): 60-68.

22. Rzany B, Hering O, Mockenhaupt M, Goerttler E, Ring J, et al. (1996) Histopathological and epidemiological characteristics of patients with erythema exudativum multiforme major, Stevens-Johnson syndrome and toxic epidermal necrolysis. Br J Dermatol 135(1): 6-11.

23. Mayorga C, Sanz ML, Gamboa P, García-Avilés MC,
Fernández J, et al. (2013) In vitro methods for diagnosing nonimmediate hypersensitivity reactions to drugs. J Allergy Clin Immunol 23(4): 213- 225.

24. Garcia-Doval I, LeCleach L, Bocquet H, Otero XL, Roujeau JC, et al. (2000) Toxic epidermal necrolysis and StevensJohnson syndrome: does early with- drawal of causative drugs decrease the risk of death? Arch Dermatol 136(3): 323-327.

25. Stern RS, Divito SJ (2017) Stevens-Johnson Syndrome and Toxic Epidermal Necrolysis: Associations, Outcomes, and Pathobiology-Thirty Years of Progress but Still Much to Be Done. Journal of Investigative Dermatology 137(5): 1004-1008.

26. Zimmermann S, Sekula P, Venhoff M, Motschall E, Knaus J, et al. (2017) Systemic Immunomodulating Therapies for Stevens-Johnson Syndrome and Toxic Epidermal Necrolysis: A Systematic Review and Meta-analysis. JAMA Dermatology 153(6): 514-522.

27. Guégan S, Bastuji-Garin S, Poszepczynska-Guigné E, Roujeau JC, Revuz J, et al. (2006) Performance of the SCORTEN during the first five days of hospitalization to predict the prognosis of epidermal necrolysis. J Invest Dermatol 126(2): 272-276.

28. Dawes J, Rowley J (2005) Enhancing the customer experience: contributions from information technology. J Business Res 36(5): 350-357.

29. Park GD, PharmD, Spector R, Headstream T (1982) Serious Adverse Reactions Associated With Sulindac. Arch Intern Med 142(7): 1292-1294.

30. Levitt L, Pearson RW (1980) Sulindac-induced StevensJohnson toxic epidermal necrolysis syndrome. JAMA 243(12): 1262-1263.

31. Husain Z, Runge LA, Jabbs JM, Hyla JA (1981) Sulindacinduced Stevens-Johnson syndrome: report of 3 cases. J Rheumatol 8(1): 176-179. 